\title{
Metallicities for six nearby open clusters from high-resolution spectra of giant stars ${ }^{\star}$
}

\section{$[\mathrm{Fe} / \mathrm{H}]$ values for a planet search sample}

\author{
N. C. Santos ${ }^{1,2}$, C. Lovis ${ }^{3}$, J. Melendez ${ }^{4}$, M. Montalto ${ }^{1}$, D. Naef ${ }^{3}$, and G. Pace ${ }^{1}$ \\ ${ }^{1}$ Centro de Astrofísica, Universidade do Porto, Rua das Estrelas, 4150-762 Porto, Portugal \\ e-mail: nuno@astro.up.pt \\ 2 Departamento de Física e Astronomia, Faculdade de Ciências, Universidade do Porto, Portugal \\ 3 Observatoire de Genève, Université de Genève, 51 ch. des Maillettes, 1290 Sauverny, Switzerland \\ 4 Departamento de Astronomia do IAG/USP, Universidade de São Paulo, Rua do Matão 1226, Cidade Universitária, \\ 05508-900 São Paulo, SP, Brasil
}

Received 14 October 2011 / Accepted 20 December 2011

\begin{abstract}
We present a study of the stellar parameters and iron abundances of 18 giant stars in six open clusters. The analysis was based on high-resolution and high-S/N spectra obtained with the UVES spectrograph (VLT-UT2). The results complement our previous study where 13 clusters were already analyzed. The total sample of 18 clusters is part of a program to search for planets around giant stars. The results show that the 18 clusters cover a metallicity range between -0.23 and +0.23 dex. Together with the derivation of the stellar masses, these metallicities will allow the metallicity and mass effects to be disentangled when analyzing the frequency of planets as a function of these stellar parameters.
\end{abstract}

Key words. planetary systems - stars: abundances - techniques: spectroscopic - open clusters and associations: general stars: fundamental parameters

\section{Introduction}

An increasing amount of evidence exists that stellar mass is a key parameter regulating giant planet formation. For instance, the frequency of giant planets orbiting (low-mass) M-dwarfs is considerably lower than the one found for FGK dwarfs (Bonfils et al. 2005; Endl et al. 2006). Higher mass stars also seem to have a higher frequency of orbiting planets (Lovis \& Mayor 2007; Johnson et al. 2007a). This result is expected from the models of planetary formation (Laughlin et al. 2004; Ida \& Lin 2005; Kennedy \& Kenyon 2008), although a consensus does not exist on this point (Kornet et al. 2005; Boss 2006).

Addressing the frequency of planets around stars of different masses is, however, not a simple task. FGK dwarfs only occupy a narrow range in mass (roughly from 0.8 to $1.2 M_{\odot}$ ), making any study of the planet frequency-stellar mass correlation difficult. For their lower mass counterparts, M-dwarfs, the radial-velocity method is often made difficult by the high stellar activity levels (e.g. Forveille et al. 2009), though this is, however, balanced by the higher amplitude signals expected. On the upper mass side main-sequence objects present a tougher case, because of the lack of spectral information (lines), together with the usually high rotation velocities of higher mass dwarfs. Although a few results exist (e.g. Galland et al. 2005), the search for planets orbiting main sequence, intermediate-mass stars has not seen much success.

\footnotetext{
* Based on observations collected at the La Silla Parana Observatory, ESO (Chile) with the UVES spectrograph at the 8.2-m Kueyen telescope, under program 383.C-0170.
}

One way to circumvent this problem is to search for planets around intermediate-mass evolved (giant or subgiant) stars. A few giant planet candidates have indeed been announced around these kind of objects (e.g. Frink et al. 2002; Sato et al. 2003; Setiawan et al. 2005; Hatzes et al. 2006; Niedzielski et al. 2007; Johnson et al. 2007b). The problem with this approach is that it is very difficult to derive precise and uniform mass values for field giants (see e.g. Lloyd 2011).

The key to solving this problem may be to search for planets orbiting clump giant stars in galactic open clusters. The position of the turnoff stars in the cluster allows setting strong constraints on their masses, giving immediate information about the mass of the clump giants. Furthermore, observing clusters with different ages (and metallicities) will allow stars to be covered within a whole range of masses. From the different surveys for such objects, three giant planets have been detected so far, namely those orbiting NGC 2423 No. 3, NGC 4349 No. 127 (Lovis \& Mayor 2007), and $\epsilon$ Tau (Sato et al. 2007).

In Santos et al. (2009, hereafter Paper I) we derived atmospheric parameters and chemical abundances for giant and dwarfs stars in 13 clusters that are being monitored with radial velocities to search for giant planets (Lovis \& Mayor 2007). In the present paper we complement this study by deriving precise stellar parameters and iron abundances in a sample of giant stars in five more clusters that are part of the same planet search program (plus one cluster in common with Paper I). In Sect. 2 we present our sample and the observations. In Sect. 3 we present the analysis of the data. We conclude with a short discussion in Sect. 4. 


\section{Sample and data}

In Paper I we presented the stellar parameters and chemical abundances for 39 giants and 16 dwarfs in the 13 open clusters IC 2714, IC 4651, IC 4756, NGC 2360, NGC 2423, NGC 2447 (M93), NGC 2539, NGC 2682 (M67), NGC 3114, NGC 3680, NGC 4349, NGC 5822, and NGC 6633. These 13 clusters are part of the 18 being surveyed for planets by Lovis \& Mayor (2007). To complement the study presented in Paper I, we present here a detailed spectroscopic analysis of 15 giants in the five remaining clusters, namely NGC 2287, NGC 2567, NGC 3532, NGC 6494, and NGC 6705. As a consistency check, three giants in NGC 2447 were also observed again, even though this cluster had already been analyzed in Paper I (using a different data set).

For each cluster, three giants were chosen based on CORALIE $^{1}$ and HARPS ${ }^{2}$ data collected as part of the planet search program (for details we refer to Lovis \& Mayor 2007). CORALIE and HARPS data allow us to assure that the giants are cluster members (their radial-velocities are compatible with membership), as well as that they are not short period binary stellar systems (e.g. spectroscopic binaries).

High-resolution UVES spectra (at the VLT-UT2 Kueyen telescope - Dekker et al. 2000) were obtained for each of the 18 giants. The observations were carried out in service mode in March 2009, under ESO program 383.C-0170. As for the data presented in Paper I, the spectra were taken in the RED580 mode. The resulting spectra cover the wavelength domain between 4780 and $6805 \AA$, with a gap between 5730 and $5835 \AA$ (corresponding to the gap in the CCD mosaic). A slit width of 0.3 arcsec was adopted, providing a resolution $R=\lambda / \Delta \lambda \approx 100000$. This is significantly higher than the one presented in Paper I $(R=50000)$. The data for NGC 2447, available in both formats, allows us to verify if using a different resolution will have any systematic effect on the derived stellar parameters and iron abundances. The $\mathrm{S} / \mathrm{N}$ of the final spectra is around 200 for all stars.

\section{Stellar parameters and iron abundances}

Stellar atmospheric parameters and iron abundances were derived in LTE using the 2002 version of the code MOOG (Sneden $1973)^{3}$ and a grid of Kurucz Atlas plane-parallel model atmospheres (Kurucz 1993). Parameters were obtained by imposing excitation and ionization equilibrium to a set of $\mathrm{Fe}_{\mathrm{I}}$ and Fe II lines, following the basic prescription described in Santos et al. (2004). In brief, the parameters were derived through an iterative process until the slope of the relation between the abundances given by individual $\mathrm{Fe}$ I lines and both the excitation potential $\left(\chi_{1}\right)$ and reduced equivalent width $(\log \mathrm{EW} / \lambda)$ were zero, and until the Fe I and Fe II lines provided the same average abundance. The adopted solar abundances are from Anders \& Grevesse (1989) except for iron, for which we adopt a value of $\log \epsilon(\mathrm{Fe})=7.47$ (the same as used in Paper I, taken from Gonzalez \& Laws 2000). Given that we are doing a differential analysis relative to the Sun, we do not expect that our results have any significant dependence on the adopted solar abundances. Individual line equivalent widths $(\mathrm{EW})$ for the iron lines

\footnotetext{
1 At the 1.2-m Euler Swiss Telescope, La Silla, Chile.

2 At the 3.6-m ESO telescope, La Silla, Chile.

${ }^{3}$ http://verdi.as.utexas.edu/moog.html
}

Table 1. Target list of UVES-VLT program 383.C-0170.

\begin{tabular}{lccc}
\hline \hline Star & $\alpha(2000.0)$ & $\delta(2000.0)$ & $V$ \\
\hline NGC 2287 & & & \\
No. 21 & $06: 45: 57.46$ & $-20: 46: 30.2$ & 6.91 \\
No. 75 & $06: 45: 43.02$ & $-20: 51: 09.6$ & 7.50 \\
No. 97 & $06: 46: 04.84$ & $-20: 36: 24.9$ & 7.78 \\
NGC 2447 & & & \\
No. 28 & $07: 44: 50.25$ & $-23: 52: 27.1$ & 9.85 \\
No. 34 & $07: 44: 33.66$ & $-23: 51: 42.2$ & 10.12 \\
No. 41 & $07: 44: 25.73$ & $-23: 49: 53.0$ & 10.03 \\
NGC 2567 & & & \\
No. 16 & $08: 18: 35.26$ & $-30: 38: 57.9$ & 11.04 \\
No. 54 & $08: 18: 26.43$ & $-30: 39: 30.4$ & 11.16 \\
No. 114 & $08: 18: 19.18$ & $-30: 32: 58.4$ & 10.87 \\
NGC 3532 & & & \\
No. 19 & $11: 05: 58.74$ & $-58: 43: 29.4$ & 7.74 \\
No. 100 & $11: 06: 03.84$ & $-58: 41: 15.8$ & 7.50 \\
No. 122 & $11: 05: 45.63$ & $-58: 40: 39.4$ & 8.20 \\
NGC 6494 & & & \\
No. 6 & $17: 56: 51.99$ & $-19: 00: 03.4$ & 9.68 \\
No. 48 & $17: 56: 23.04$ & $-19: 08: 58.7$ & 9.57 \\
No. 49 & $17: 56: 41.17$ & $-19: 08: 38.3$ & 9.70 \\
NGC 6705 & & & \\
No. 1090 & $18: 51: 03.99$ & $-06: 20: 41.0$ & 11.87 \\
No. 1184 & $18: 51: 02.02$ & $-06: 17: 26.2$ & 11.43 \\
No. 1111 & $18: 51: 03.60$ & $-06: 16: 11.0$ & 11.90 \\
\hline & & &
\end{tabular}

Notes. Data from Mermilliod \& Paunzen (2003).

were measured using the automatic ARES code (Sousa et al. 2007, 2008) $)^{4}$.

As in Paper I, the stellar parameters and iron abundances for our giant stars were derived using two different line lists. First, we used the line list described in Sousa et al. (2008, hereafter S08), composed of 263 Fe I and 36 Fe II lines in the optical domain. The use of this line list has shown to give excellent results for the analysis of dwarf stars (Sousa et al. 2008). Beyond this, we also derived the stellar parameters using the line list provided by Hekker \& Meléndez (2007, hereafter HM07). Although much shorter (20 Fe I and $6 \mathrm{Fe}$ II lines), the lines in this list were carefully chosen for the analysis of giant stars, avoiding lineblending from CN lines (Meléndez \& Barbuy 1999).

As shown in Paper I, the parameters derived using the HM07 line list may be preferable since they provide metallicities on the same scale as the ones derived for dwarf stars. However, since they are more subject to analysis errors (much shorter line list), in Paper I we proposed a correction for the $[\mathrm{Fe} / \mathrm{H}]$ values derived using the S08 line-list. This correction puts the derived metallicities for the giant stars on the same scale as those found for dwarfs of the same cluster. For that we used the average metallicities derived from dwarfs and giants in six different clusters, spanning a metallicity range roughly between -0.1 and +0.2 dex (see also Fig. 1, left panel).

The derived (uncorrected) stellar parameters and iron abundances for all the stars analyzed in this paper are listed in Table 2, together with the number of Fe I and Fe II lines used and the rms of their individual abundances. The cluster-averaged metallicity values can be seen in Table 3, where we list the values derived using both the S08 and the HM07 line lists. The corrected metallicities, $\langle[\mathrm{Fe} / \mathrm{H}]\rangle_{\mathrm{S} 08}^{\mathrm{c}}$, refer to the values derived using the line list

${ }^{4}$ http://www.astro.up.pt/ sousasag/ares/ 

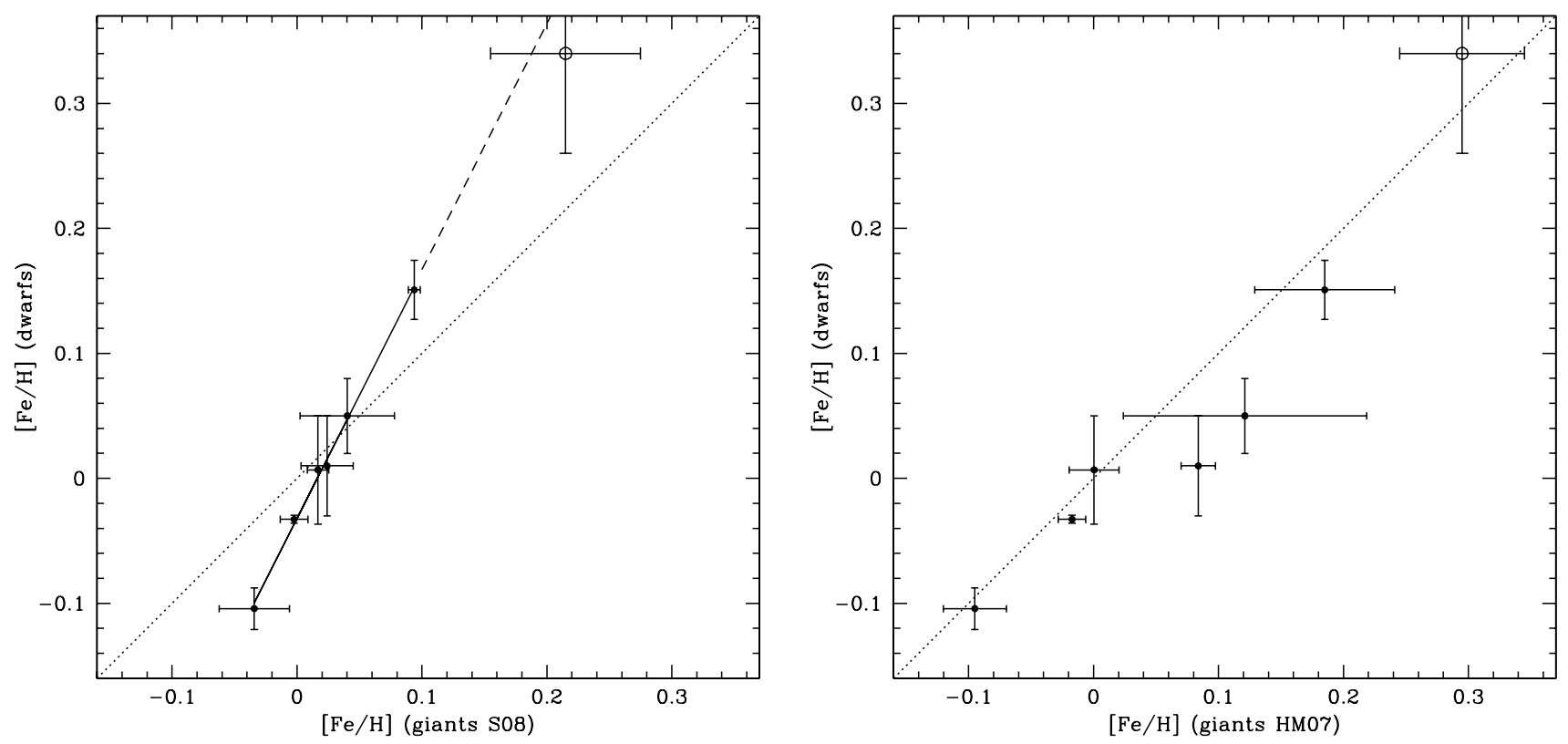

Fig. 1. Comparison of the average values for the metallicities derived from dwarfs and giants in the 6 clusters from Paper I (dots), as well as for dwarfs and giants from NGC 6253 (Montalto et al. 2012, open circle). See text for more details.

of S08 and subsequently corrected using the relation presented in Paper I (illustrated in Fig. 1, left panel - see discussion in Sect. 3.1). We adopt these metallicities as our final values.

For some of the stars in our sample, the small number of lines in the HM07 line list did not allow us to derive reliable parameters (e.g. measurable lines in the spectra did not cover a wide enough excitation potential range). This was the case for stars No. 21 and No. 1090 in NGC 2287 and NGC 6705, respectively.

For some of the stars we derived effective temperature values below $4600 \mathrm{~K}$. These are the cases of NGC 2287 No. 21 and NGC 6705 No. 1184 (using the S08 line list) and NGC 2287 No. 75 and NGC 6705 No. 1184 (using the HM07 line-list). Since we are not sure that the model atmospheres used are valid for such low temperatures and considering that line blending is more severe at lower temperatures, we decided to keep these stars out of the sample when computing the average abundances in these clusters (Table 3 ). When one single star is left, the value in the table denotes the metallicity (and error) derived for this star

Finally, we note that for NGC 2287, the metallicity found using the S08 line list puts this cluster outside the range for which the correction was derived (see Fig. 1 and Sect.3.1). The obtained $\langle[\mathrm{Fe} / \mathrm{H}]\rangle_{\mathrm{S} 08}^{\mathrm{c}}$ value must in this case be taken with care. As described in Sect. 3.1, while for high metallicities the results for NGC 6253 seem to confirm that the calibration is valid in the high- $[\mathrm{Fe} / \mathrm{H}]$ regime, we have no confirmation for lower metallicity values.

\subsection{The metallicity scale for giants and dwarfs}

In Fig. 1 we show two plots similar to those presented in Fig. 1 of Paper I, where we compare the metallicities derived for the dwarfs and giants in the six clusters presented in that paper. Additionally, in the plot we include the metallicities derived for NGC 6253, a metal rich cluster whose abundances were recently derived by our team and presented in Montalto et al. (2012) ${ }^{5}$. In both panels, the dotted line represents the 1:1 relation, while the solid line in the left panel represents a linear fit to the filled points, with a relation $\langle[\mathrm{Fe} / \mathrm{H}]\rangle_{\text {dwarfs }}=1.97\langle[\mathrm{Fe} / \mathrm{H}]\rangle_{\mathrm{S} 08}-0.03$ (see Paper I). The dashed line represents the extrapolation of this fit to higher metallicities. S08 and HM07 refer to the abundances derived using the line lists of Sousa et al. (2008) and Hekker \& Meléndez (2007), respectively.

As can be seen from Fig. 1, the position of NGC 6253 seems to confirm our previous finding that the S08 line list, despite its adequacy for the study of dwarf stars (Sousa et al. 2008), does not deliver $[\mathrm{Fe} / \mathrm{H}]$ values on the same scale for dwarfs and giant stars. On the other hand, the HM07 line list seems adequate for studying giants. The calibration proposed in Paper I to correct the metallicities derived using the S08 line list also seems to be appropriate. We decided not to change this calibration using the new values for NGC 6253 since only one dwarf has been analyzed in our study of this cluster (Montalto et al. 2012).

The cause of the offset metallicity values found using the S08 line list is not clear. In Paper I we suggested that it could be because (cool) giant stars have higher macroturbulence velocities (Gray 1992) and because thousands of molecular lines $(\mathrm{CN}, \mathrm{C} 2, \mathrm{CH}, \mathrm{MgH})$ contribute to the optical spectra of giants (Coelho et al. 2005). These effects lead to line-blending, which is stronger for higher metallicity and cooler objects. Indeed, in the present work the coolest giants $(<4600 \mathrm{~K})$ seem more metal-poor than the hottest giants in the same cluster (see results for NGC 2287 and NGC 6705), which could be explained by severe line crowding, leading to underestimating the true continuum and therefore to underestimating their metallicities. Interestingly, the problem with the metallicity scale for giants (with respect to dwarfs) has also been recognized by other

5 The values derived are: for the only dwarf analyzed, $[\mathrm{Fe} / \mathrm{H}]=+0.34 \pm 0.08$; for the two giants analyzed, the average metallicities and their rms are $+0.22 \pm 0.06$ and $+0.30 \pm 0.05$, respectively using the S08 and HM07 line lists. 
Table 2. Stellar parameters derived for the giants stars.

\begin{tabular}{|c|c|c|c|c|c|c|}
\hline Star & $\begin{array}{l}T_{\text {eff }} \\
{[\mathrm{K}]}\end{array}$ & $\begin{array}{c}\log g_{\text {spec }} \\
{\left[\mathrm{g} \text { in } \mathrm{cm} \mathrm{s}^{-2}\right]}\end{array}$ & $\begin{array}{c}\xi_{\mathrm{t}} \\
{\left[\mathrm{km} \mathrm{s}^{-1}\right]}\end{array}$ & {$[\mathrm{Fe} / \mathrm{H}]$} & $\mathrm{N}\left(\mathrm{Fe}_{\mathrm{I}}, \mathrm{Fe}\right.$ II $)$ & $\sigma\left(\mathrm{Fe}_{\mathrm{I}}, \mathrm{Fe}_{\mathrm{II}}\right)$ \\
\hline \multicolumn{7}{|c|}{ Results using the S08 line-list: } \\
\hline NGC 2287 No. $21^{\dagger}$ & $4350 \pm 82$ & $1.71 \pm 0.66$ & $2.49 \pm 0.08$ & $-0.24 \pm 0.24$ & 179,25 & $0.24,0.37$ \\
\hline NGC 2287 No. 75 & $4617 \pm 57$ & $2.03 \pm 0.37$ & $2.20 \pm 0.05$ & $-0.12 \pm 0.18$ & 179,24 & $0.18,0.20$ \\
\hline NGC 2287 No. 97 & $4764 \pm 46$ & $2.15 \pm 0.40$ & $1.98 \pm 0.04$ & $-0.09 \pm 0.14$ & 181,25 & $0.14,0.20$ \\
\hline NGC 2447 No. 28 & $5143 \pm 28$ & $2.76 \pm 0.26$ & $1.61 \pm 0.03$ & $-0.05 \pm 0.09$ & 180,25 & $0.09,0.12$ \\
\hline NGC 2447 No. 34 & $5242 \pm 35$ & $3.01 \pm 0.29$ & $1.63 \pm 0.03$ & $0.03 \pm 0.11$ & 179,25 & $0.11,0.13$ \\
\hline NGC 2447 No. 41 & $5215 \pm 25$ & $2.94 \pm 0.36$ & $1.59 \pm 0.02$ & $0.00 \pm 0.08$ & 179,25 & $0.08,0.16$ \\
\hline NGC 2567 No. 16 & $5205 \pm 42$ & $2.82 \pm 0.36$ & $1.59 \pm 0.04$ & $-0.04 \pm 0.14$ & 182,23 & $0.13,0.16$ \\
\hline NGC 2567 No. 54 & $5216 \pm 36$ & $2.89 \pm 0.34$ & $1.73 \pm 0.04$ & $0.06 \pm 0.12$ & 182,23 & $0.12,0.16$ \\
\hline NGC 2567 No. 114 & $5078 \pm 39$ & $2.73 \pm 0.33$ & $1.89 \pm 0.04$ & $0.01 \pm 0.14$ & 180,24 & $0.13,0.16$ \\
\hline NGC 3532 No. 19 & $5089 \pm 34$ & $2.69 \pm 0.22$ & $1.66 \pm 0.03$ & $0.05 \pm 0.11$ & 182,25 & $0.11,0.10$ \\
\hline NGC 3532 No. 100 & $4938 \pm 41$ & $2.54 \pm 0.21$ & $1.85 \pm 0.04$ & $0.00 \pm 0.13$ & 179,24 & $0.13,0.10$ \\
\hline NGC 3532 No. 122 & $5218 \pm 55$ & $3.09 \pm 0.71$ & $1.74 \pm 0.06$ & $0.01 \pm 0.17$ & 178,25 & $0.16,0.31$ \\
\hline NGC 6494 No. 6 & $4926 \pm 42$ & $2.50 \pm 0.24$ & $1.90 \pm 0.04$ & $0.02 \pm 0.14$ & 181,24 & $0.13,0.12$ \\
\hline NGC 6494 No. 48 & $5131 \pm 46$ & $2.71 \pm 0.46$ & $1.86 \pm 0.05$ & $0.04 \pm 0.16$ & 179,24 & $0.15,0.21$ \\
\hline NGC 6494 No. 49 & $5012 \pm 48$ & $2.63 \pm 0.36$ & $1.82 \pm 0.04$ & $0.06 \pm 0.16$ & 181,25 & $0.15,0.17$ \\
\hline NGC 6705 No. 1090 & $4844 \pm 70$ & $2.43 \pm 0.6$ & $2.06 \pm 0.06$ & $0.12 \pm 0.22$ & 181,24 & $0.21,0.31$ \\
\hline NGC 6705 No. 1111 & $5039 \pm 74$ & $2.85 \pm 0.54$ & $2.18 \pm 0.08$ & $0.14 \pm 0.24$ & 184,24 & $0.23,0.27$ \\
\hline NGC 6705 No. $1184^{\dagger}$ & $4518 \pm 65$ & $2.09 \pm 0.42$ & $1.92 \pm 0.06$ & $-0.01 \pm 0.19$ & 178,23 & $0.19,0.23$ \\
\hline \multicolumn{7}{|c|}{ Results using the HM07 line-list: } \\
\hline NGC 2287 No. $75^{\dagger}$ & $4381 \pm 135$ & $1.73 \pm 0.33$ & $2.11 \pm 0.15$ & $-0.20 \pm 0.17$ & 16,6 & 0.07 \\
\hline NGC 2287 No. 97 & $4638 \pm 84$ & $2.14 \pm 0.20$ & $1.87 \pm 0.10$ & $-0.11 \pm 0.11$ & 15,6 & $0.09,0.07$ \\
\hline NGC 2447 No. 28 & $5077 \pm 97$ & $2.90 \pm 0.14$ & $1.71 \pm 0.18$ & $-0.08 \pm 0.14$ & 15,6 & $0.10,0.03$ \\
\hline NGC 2447 No. 34 & $5064 \pm 95$ & $2.90 \pm 0.17$ & $1.76 \pm 0.22$ & $-0.10 \pm 0.16$ & 16,6 & $0.12,0.06$ \\
\hline NGC 2447 No. 41 & $5109 \pm 75$ & $2.72 \pm 0.40$ & $1.60 \pm 0.15$ & $-0.07 \pm 0.12$ & 16,6 & $0.09,0.19$ \\
\hline NGC 2567 No. 16 & $5061 \pm 85$ & $2.72 \pm 0.24$ & $1.67 \pm 0.19$ & $-0.17 \pm 0.14$ & 16,6 & $0.10,0.11$ \\
\hline NGC 2567 No. 54 & $5040 \pm 93$ & $2.66 \pm 0.30$ & $1.73 \pm 0.16$ & $-0.04 \pm 0.15$ & 16,6 & $0.11,0.14$ \\
\hline NGC 2567 No. 114 & $4890 \pm 98$ & $2.59 \pm 0.21$ & $1.86 \pm 0.14$ & $-0.09 \pm 0.14$ & 16,6 & $0.10,0.08$ \\
\hline NGC 3532 No. 19 & $4944 \pm 66$ & $2.47 \pm 0.19$ & $1.57 \pm 0.10$ & $0.00 \pm 0.11$ & 16,6 & $0.08,0.09$ \\
\hline NGC 3532 No. 100 & $4766 \pm 74$ & $2.22 \pm 0.22$ & $1.71 \pm 0.09$ & $-0.07 \pm 0.12$ & 15,6 & $0.09,0.10$ \\
\hline NGC 3532 No. 122 & $4929 \pm 81$ & $2.51 \pm 0.51$ & $1.41 \pm 0.14$ & $-0.14 \pm 0.13$ & 15,6 & $0.10,0.25$ \\
\hline NGC 6494 No. 6 & $4779 \pm 76$ & $2.27 \pm 0.15$ & $1.78 \pm 0.10$ & $-0.07 \pm 0.11$ & 15,6 & $0.08,0.04$ \\
\hline NGC 6494 No. 48 & $4909 \pm 84$ & $2.54 \pm 0.18$ & $1.90 \pm 0.17$ & $-0.14 \pm 0.13$ & 16,6 & $0.10,0.07$ \\
\hline NGC 6494 No. 49 & $4795 \pm 107$ & $2.26 \pm 0.32$ & $1.80 \pm 0.14$ & $-0.11 \pm 0.15$ & 16,6 & $0.11,0.14$ \\
\hline NGC 6705 No. 1111 & $4685 \pm 190$ & $2.28 \pm 0.52$ & $2.26 \pm 0.28$ & $-0.06 \pm 0.25$ & 16,6 & $0.19,0.22$ \\
\hline NGC 6705 No. $1184^{\dagger}$ & $4288 \pm 88$ & $1.72 \pm 0.31$ & $1.96 \pm 0.09$ & $-0.14 \pm 0.09$ & 15,6 & $0.08,0.13$ \\
\hline
\end{tabular}

Notes. See text for more details. ${ }^{(\dagger)}$ Given their low derived effective temperatures, these measurements were excluded when computing the metallicities listed in Table 3.

Table 3. Weighted average metallicities of the giant stars in each of the 18 clusters (analysed in this paper and in Paper I).

\begin{tabular}{lrrrl}
\hline \hline Cluster & $\langle[\mathrm{Fe} / \mathrm{H}]\rangle_{\mathrm{S} 08}$ & \multicolumn{1}{c}{$\langle[\mathrm{Fe} / \mathrm{H}]\rangle_{\mathrm{S} 08}^{\mathrm{c}}$} & \multicolumn{1}{c}{$\langle[\mathrm{Fe} / \mathrm{H}]\rangle_{\mathrm{HM} 07}$} & Souce \\
\hline IC 2714 & $0.02 \pm 0.01$ & $0.01 \pm 0.01$ & $-0.03 \pm 0.04$ & Paper I \\
IC 4651 & $0.09 \pm 0.01$ & $0.15 \pm 0.01$ & $0.19 \pm 0.06$ & Paper I \\
IC 4756 & $0.02 \pm 0.02$ & $0.02 \pm 0.02$ & $0.08 \pm 0.01$ & Paper I \\
NGC 2360 & $0.00 \pm 0.01$ & $-0.03 \pm 0.01$ & $-0.01 \pm 0.03$ & Paper I \\
NGC 2423 & $0.09 \pm 0.06$ & $0.14 \pm 0.06$ & $0.07 \pm 0.06$ & Paper I \\
NGC 2447 & $-0.03 \pm 0.03$ & $-0.10 \pm 0.03$ & $-0.10 \pm 0.03$ & Paper I \\
NGC 2539 & $0.08 \pm 0.03$ & $0.13 \pm 0.03$ & $0.09 \pm 0.02$ & Paper I \\
NGC 2682 & $0.02 \pm 0.01$ & $0.00 \pm 0.01$ & $0.00 \pm 0.02$ & Paper I \\
NGC 3114 & $0.02 \pm 0.09$ & $0.02 \pm 0.09$ & $0.00 \pm 0.12$ & Paper I \\
NGC 3680 & $0.00 \pm 0.01$ & $-0.04 \pm 0.01$ & $-0.02 \pm 0.01$ & Paper I \\
NGC 4349 & $-0.04 \pm 0.06$ & $-0.12 \pm 0.06$ & $-0.06 \pm 0.08$ & Paper I \\
NGC 5822 & $0.04 \pm 0.04$ & $0.05 \pm 0.04$ & $0.12 \pm 0.10$ & Paper I \\
NGC 6633 & $0.04 \pm 0.01$ & $0.06 \pm 0.01$ & $0.00 \pm 0.00$ & Paper I \\
\hline NGC 2287 & $-0.10 \pm 0.02$ & $-0.23 \pm 0.02$ & $-0.11 \pm 0.11$ & This paper \\
NGC 2447 & $-0.01 \pm 0.04$ & $-0.05 \pm 0.04$ & $-0.08 \pm 0.01$ & This paper \\
NGC 2567 & $0.01 \pm 0.05$ & $0.00 \pm 0.05$ & $-0.10 \pm 0.06$ & This paper \\
NGC 3532 & $0.03 \pm 0.03$ & $0.02 \pm 0.03$ & $-0.06 \pm 0.07$ & This paper \\
NGC 6494 & $0.04 \pm 0.02$ & $0.04 \pm 0.02$ & $-0.10 \pm 0.04$ & This paper \\
NGC 6705 & $0.13 \pm 0.01$ & $0.23 \pm 0.01$ & $-0.06 \pm 0.25$ & This paper \\
\hline
\end{tabular}

Notes. ${ }^{\dagger}$ For this cluster we adopt the average value of the two derivations. 


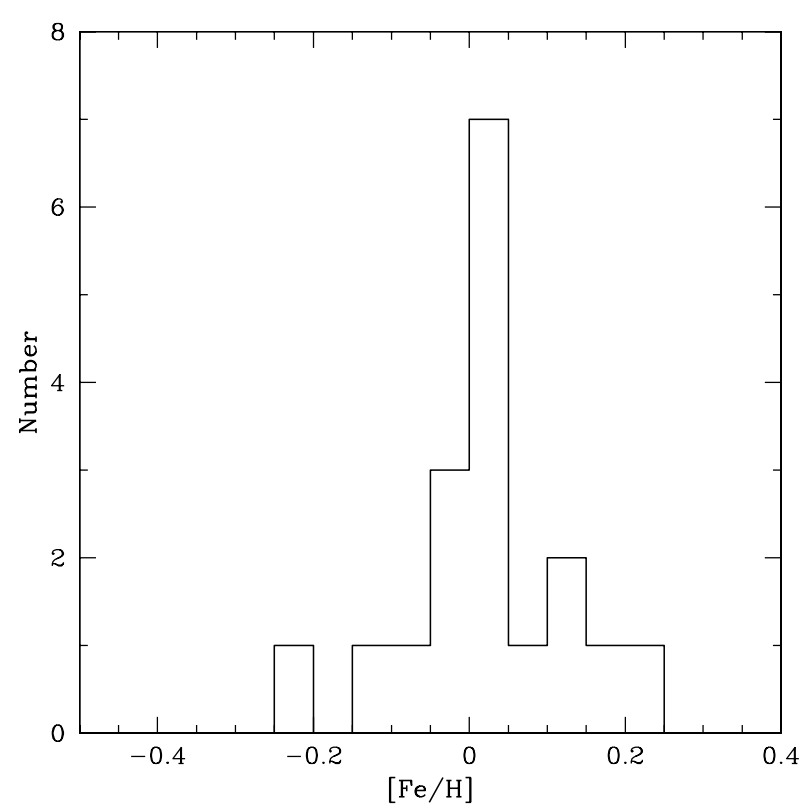

Fig. 2. Metallicity distribution of the average metallicities for the 18 clusters in Table 3 using the corrected S08 values, $\langle[\mathrm{Fe} / \mathrm{H}]\rangle_{\mathrm{S} 08}^{\mathrm{c}}$.

studies of giants in the solar neighborhood (Taylor \& Croxall 2005), as well as of bulge stars (e.g. Cohen et al. 2008).

Using higher resolution spectra could in principle minimize this problem. However, comparison of the results for NGC 2447, whose spectra in Paper I were taken with a resolution of $\approx 50000$, while here we use data with a resolution of $\approx 100000$ (for the very same stars), does not confirm this possibility. Both sets of spectra provide very similar results in terms of stellar metallicity (see the $\langle[\mathrm{Fe} / \mathrm{H}]\rangle_{\mathrm{S} 08}$ and $\langle[\mathrm{Fe} / \mathrm{H}]\rangle_{\mathrm{HM} 07}$ values in Table 3), although the small observed difference goes in the right direction: slightly higher metallicities are obtained using spectra of higher resolution (even if the values are compatible within the errors). On the other hand, this gives us confidence that the results are homogeneous and can be treated together. For the remainder of this paper we use the average metallicity values $\left(\langle[\mathrm{Fe} / \mathrm{H}]\rangle_{\mathrm{S} 08}^{\mathrm{c}}=-0.07 \mathrm{dex}\right)$ derived in the two papers for this cluster.

To test whether a wrong estimate of the surface gravities could be the cause of the observed discrepancy, we tested the influence of fixing $\log g$ to $2.50 \mathrm{dex}$ in NGC 2447 No. 41 i.e., $\approx 0.5$ dex below the value obtained using the S08 line list. From this procedure we determined values of $5252 \mathrm{~K}, 1.67 \mathrm{~km} \mathrm{~s}^{-1}$, and 0.00 dex for the derived effective temperature, microturbulence, and stellar metallicity respectively. The variations with respect to the value listed in Table 2 are always within the error bars, and the derived metallicity did not suffer any change.

\section{Discussion}

In Fig. 2 we present the metallicity distribution for the 13 clusters studied in Paper I, together with the five more clusters presented in this paper. Their metallicities (we are considering the $\langle[\mathrm{Fe} / \mathrm{H}]\rangle_{\mathrm{S} 08}^{\mathrm{c}}$ values) range from -0.23 to $+0.23 \mathrm{dex}$, with a peak near solar values. Most of the clusters seem, however, to have metallicities above solar (only 6 among the 18 have $[\mathrm{Fe} / \mathrm{H}]<0$ ).
The knowledge about the metallicity of these clusters is of utmost importance for interpretating the planet search results. It is well known that the metallicity of the star is a prime factor in determining the frequency of giant planets (Santos et al. 2001, 2004; Fischer \& Valenti 2005; Sousa et al. 2011). Present results suggest that the observed metallicity-giant planet correlation reflects the higher probability of forming planets orbiting metal-rich stars (e.g. Ida \& Lin 2004; Mordasini et al. 2009).

This possibility has been put in question by the suggestion that the metallicity-giant planet correlation may not hold for intermediate-mass (giant) stars hosting giant planets (Pasquini et al. 2007; Ghezzi et al. 2010). Although not fully accepted (Hekker \& Meléndez 2007), this possible lack of correlation could hint that stellar mass strongly influences the planet formation process (Laughlin et al. 2004; Ida \& Lin 2005; Kennedy $\&$ Kenyon 2008). In either case, since both mass and metallicity seem to influence the planet formation process, the knowledge about the stellar metallicity is crucial if one wants to disentangle both effects in the analysis of the results from the planet search program.

Since the number of stars surveyed in each cluster is not very high (a dozen stars on average - Lovis \& Mayor 2007), knowledge of their metallicity may also constrain which clusters are more likely to harbor planets, and thus focus the efforts of the survey. Alternatively, considering the metallicity-giant planet correlation observed for dwarfs, it may be also wise to increase the sample in the less metal-rich clusters.

Until the present date, two giant planets have been announced as orbiting stars from the surveyed clusters: NGC 2423 No. 3 and NGC 4349 No. 127 (Lovis \& Mayor 2007). The two clusters have average metallicities of $+0.14 \pm 0.06$ and $-0.12 \pm 0.06$, respectively (using the corrected values in Table 3). Such a small number of planets preclude a statistical analysis of these results. However, it is curious to see that these two clusters are among the most metal-rich (NGC 2423) and metal-poor (NGC 4349) populations in the sample.

Acknowledgements. This work was supported by the European Research Council/European Community under the FP7 through Starting Grant agreement number 239953. N.C.S. also acknowledges the support from Fundação para a Ciência e a Tecnologia (FCT) through program Ciência 2007 funded by FCT/MCTES (Portugal) and POPH/FSE (EC), and in the form of grant reference PTDC/CTE-AST/098528/2008. M.M. also acknowledges the support from FCT in the form of fellowship reference SFRH/BDP/71230/2010. This research made use of the WEBDA database, operated at the Institute for Astronomy of the University of Vienna.

\section{References}

Anders, E., \& Grevesse, N. 1989, Geochim. Cosmochim. Acta, 53, 197 Bonfils, X., Forveille, T., Delfosse, X., et al. 2005, A\&A, 443, L15 Boss, A. P. 2006, ApJ, 644, L79

Coelho, P., Barbuy, B., Meléndez, J., Schiavon, R. P., \& Castilho, B. V. 2005, A\&A, 443, 735

Cohen, J. G., Huang, W., Udalski, A., Gould, A., \& Johnson, J. A. 2008, ApJ, 682, 1029

Dekker, H., D’Odorico, S., Kaufer, A., Delabre, B., \& Kotzlowski, H. 2000, in SPIE Conf. Ser. 4008, ed. M. Iye, \& A. F. Moorwood, 534

Endl, M., Cochran, W. D., Kürster, M., et al. 2006, ApJ, 649, 436

Fischer, D. A., \& Valenti, J. 2005, ApJ, 622, 1102

Forveille, T., Bonfils, X., Delfosse, X., et al. 2009, A\&A, 493, 645

Frink, S., Mitchell, D. S., Quirrenbach, A., et al. 2002, ApJ, 576, 478

Galland, F., Lagrange, A.-M., Udry, S., et al. 2005, A\&A, 444, L21

Ghezzi, L., Cunha, K., Schuler, S. C., \& Smith, V. V. 2010, ApJ, 725, 721

Gonzalez, G., \& Laws, C. 2000, AJ, 119, 390

Gray, D. F. 1992, The Observation and Analysis of Stellar Photospheres (Cambridge University Press)

Hatzes, A. P., Cochran, W. D., Endl, M., et al. 2006, A\&A, 457, 335

Hekker, S., \& Meléndez, J. 2007, A\&A, 475, 1003 
A\&A 538, A151 (2012)

Ida, S., \& Lin, D. N. C. 2004, ApJ, 616, 567

Ida, S., \& Lin, D. N. C. 2005, ApJ, 626, 1045

Johnson, J. A., Butler, R. P., Marcy, G. W., et al. 2007a, ApJ, 670, 833

Johnson, J. A., Fischer, D. A., Marcy, G. W., et al. 2007b, ApJ, 665, 785

Kennedy, G. M., \& Kenyon, S. J. 2008, ApJ, 673, 502

Kornet, K., Bodenheimer, P., Różyczka, M., \& Stepinski, T. F. 2005, A\&A, 430, 1133

Kurucz, R. 1993, R. Kurucz CD-ROM No. 13 (Cambridge, Mass.: Smithsonian Astrophysical Observatory), 13

Laughlin, G., Bodenheimer, P., \& Adams, F. C. 2004, ApJ, 612, L73

Lloyd, J. P. 2011, ApJ, 739, L49

Lovis, C., \& Mayor, M. 2007, A\&A, 472, 657

Meléndez, J., \& Barbuy, B. 1999, ApJS, 124, 527

Mermilliod, J.-C., \& Paunzen, E. 2003, A\&A, 410, 511

Montalto, M., Santos, N., Villanova, S., et al. 2012, MNRAS, submitted

Mordasini, C., Alibert, Y., \& Benz, W. 2009, A\&A, 501, 1139
Niedzielski, A., Konacki, M., Wolszczan, A., et al. 2007, ApJ, 669, 1354 Pasquini, L., Döllinger, M. P., Weiss, A., et al. 2007, A\&A, 473, 979

Santos, N. C., Israelian, G., \& Mayor, M. 2001, A\&A, 373, 1019

Santos, N. C., Israelian, G., \& Mayor, M. 2004, A\&A, 415, 1153

Santos, N. C., Lovis, C., Pace, G., Melendez, J., \& Naef, D. 2009, A\&A, 493, 309

Sato, B., Ando, H., Kambe, E., et al. 2003, ApJ, 597, L157

Sato, B., Izumiura, H., Toyota, E., et al. 2007, ApJ, 661, 527

Setiawan, J., Rodmann, J., da Silva, L., et al. 2005, A\&A, 437, L31

Sneden, C. 1973, Ph.D. Thesis, Austin, Univ. of Texas

Sousa, S. G., Santos, N. C., Israelian, G., Mayor, M., \& Monteiro, M. J. P. F. G. 2007, A\&A, 469, 783

Sousa, S. G., Santos, N. C., Mayor, M., et al. 2008, A\&A, 487, 373

Sousa, S. G., Santos, N. C., Israelian, G., Mayor, M., \& Udry, S. 2011, A\&A, 533, A141

Taylor, B. J., \& Croxall, K. 2005, MNRAS, 357, 967 